\title{
INFLUENCE OF MEMBERSHIP FUNCTION'S SHAPE ON PORTFOLIO OPTIMIZATION RESULTS
}

\author{
Aleksandra Rutkowska \\ Department of Applied Mathematics \\ Poznan University of Economics and Business \\ al. Niepodleglosci, 61-875 Poznan, Poland
}

\begin{abstract}
Portfolio optimization, one of the most rapidly growing field of modern finance, is selection process, by which investor chooses the proportion of different securities and other assets to held. This paper studies the influence of membership function's shape on the result of fuzzy portfolio optimization and focused on portfolio selection problem based on credibility measure. Four different shapes of the membership function are examined in the context of the most popular optimization problems: mean-variance, mean-semivariance, entropy minimization, value-at-risk minimization. The analysis takes into account both: the study of necessary and sufficient conditions for the existence of extremes, as well as the statistical inference about the differences based on simulation.
\end{abstract}

Keywords: fuzzy variable, membership function, fuzzy portfolio optimization

\section{Introduction}

Portfolio optimization problem since the publication of the Markowitz [1] portfolio analysis is the basis of modern finances and a widely discussed area. By portfolio we understand the securities set held by an investor. Portfolio selection is the process by which one chooses the securities, derivatives, and other assets to include in a portfolio and their proportion in whole portfolio. In making securities selections, one considers the risk, the return, the ethical implications, and other factors affecting both of the individual securities and the portfolio as a whole. Traditionally, the asset's rate of returns are assumed to be random variables, but in the 90's of the last century optimization methods based on fuzzy logic spread widely, which assume the return rates to be fuzzy variables. Since 2002, when the credibility theory was created [2], its numerous dynamic models of portfolio optimization have developed. In 2005 [3] the first models were proposed: a mean-variance model, a model of op- timistic values and a maximum credibility model. In 2006, Huang developed a credibility maximization model and a chance-constrained programming model [4], next a mean-variance model was extended to a mean-semivariance model [5]. Then it was proposed to reduce the risk using entropy of fuzzy variable [6]. Model mean-variance has evolved in a minimax mean-variance model [7] and a mean-variance-skewness model [9]. A review of fuzzy portfolio optimization models including credibility models can be found in [8].

One of the problems in the practical implementation of these methods is the lack of ways to determine fuzzy returns rates. Considering various methods raises a question of what shape should a membership function be. Research on the influence of membership function shapes is discussed in the area of fuzzy controls. Koprinkova [10], Marshall [11], Multani [12], among others, considered it from different points of view. The first two works conclude that nonlinear MFs i.e. Gaussian are better choice 
than piecewise linear functions. The third one highlights that a membership function optimized for a certain event will unlikely perform well under various different conditions. It is difficult to find a similar analysis of the literature on fuzzy portfolio optimization. A problem considered this issue can be crucial for the returns of the selected portfolios beyond that use more complex types of fuzzy numbers can lead to an increase in computational complexity and hence the cost utilization optimization model in practice. In the case of portfolio optimization study empirical research on real market data are widespread. But to isolate affects the shape of fuzzy number on the result of the selection, we need to use simulation. Otherwise we are unable to say whether the better/worse outcome of particular portfolio can be explained by the change in shape, economic events or market inefficiency. Thus, the analysis is limited to the comparison of the distance between vectors and examines the relevance of these differences. The model present in literature use mostly triangular fuzzy numbers. Therefore, the purpose of this article is answer the question whether a triangular shape is a good approximation of other shapes. This article will check whether this is a good approximation of possible shapes and if the shape of membership function has a significant impact on the results of the portfolio optimization. The study covers four different membership functions' shapes in the context of the most popular optimization problems. This paper is organized as follows: Section 2 presents basic information about fuzzy variable and fuzzy credibilistic portfolio optimization model is presented. Section 3 formulates the problem and presents the steps of survey. Section 4 discusses the results. Section 5 covers the conclusions.

The study has been prepared as a part of the research project of the National Science Center 2013/09/N/HS4/03761 using Infrastructures PLGrid.

\section{Preliminaries}

To express uncertainty of future returns of assets fuzzy variables are used. In this section brief reviews of some basic concepts of fuzzy variable and portfolio selection models are presented within the framework of credibility theory. Credibility theory and details of fuzzy variable can be found in [13].

\subsection{Fuzzy variable}

Let $\Theta$ be a non-empty set, and $P$ the power of set $\Theta$. Each element in $P$ is called an event and $C r$ a credibility function, which indicates the credibility that an event will occur.

A fuzzy variable is defined in [2] as a function from a credibility space $(\Theta, P, C r)$ to the set of real numbers.

In this paper the following shortened term will be used: $(\xi \in B)=(\theta \in \Theta \mid \xi(\theta) \in B)$. Suppose $\xi$ is a fuzzy variable with membership function $\mu_{\xi}$ and $x$ is a real number. For any $B \in \mathfrak{R}$ and $B^{c} \in \mathfrak{R}$, where $B^{c}$ is complement of the set $B$ the credibility measure of event $\{\xi \in B\}$ was defined by Liu and Liu [2] as follows:

$$
\begin{gathered}
C r\{\xi \in B\}=\frac{1}{2}\left(\sup _{x \in B} \mu(x)+1-\sup _{x \in B^{c}} \mu(x)\right), \\
\forall x \in \mathfrak{R}
\end{gathered}
$$

In this paper we will consider fuzzy variables with four different membership functions (see Fig.1):

1. triangular

$$
\mu_{\xi}=\left\{\begin{array}{cc}
\frac{x-a}{b-a}, & \text { for } a \leq x<b \\
\frac{x-c}{b-c}, & \text { for } b \leq x<c \\
0 & \text { otherwise }
\end{array}\right.
$$

2. parabolic

$$
\mu_{\xi}=\left\{\begin{array}{cc}
1-\left(\frac{x-b}{b-a}\right)^{2}, & \text { for } a \leq x<b \\
1-\left(\frac{x-b}{c-b}\right)^{2}, & \text { for } b \leq x<c \\
0, & \text { otherwise }
\end{array}\right.
$$

3. normal $^{1}$

$$
\mu_{\xi}=\left\{\begin{array}{cc}
\left(\frac{x-a}{b-a}\right)^{2}, & \text { for } a \leq x<b \\
\left(\frac{x-c}{b-c}\right)^{2}, & \text { for } b \leq x<c \\
0, & \text { otherwise }
\end{array}\right.
$$

4. SZ type

$$
\mu_{\xi}=\left\{\begin{array}{cc}
2\left(\frac{x-a}{b-a}\right)^{2}, & \text { for } a \leq x<\frac{a+b}{2} \\
1-2\left(\frac{x-b}{b-a}\right)^{2}, & \text { for } \frac{a+b}{2} \leq x<b \\
1-2\left(\frac{x-b}{c-b}\right)^{2}, & \text { for } b \leq x<\frac{b+c}{2} \\
2\left(\frac{x-c}{c-b}\right)^{2}, & \text { for } \frac{b+c}{2} \leq x<c \\
0, & \text { otherwise }
\end{array}\right.
$$

\footnotetext{
${ }^{1}$ here the term normal refers to the shape of the membership function according to [13]. In the literature, the term normal fuzzy set refers also to a situation where the membership function of a set assumes a value between 0 and 1 , including 1 .
} 

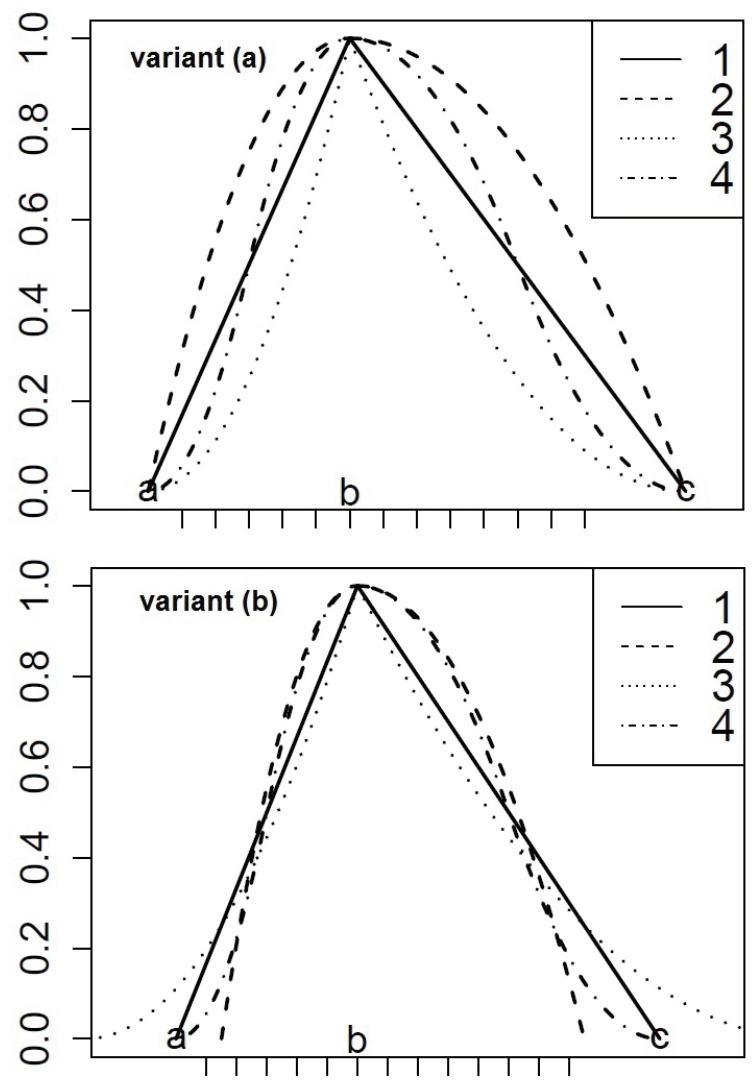

Figure 1. Shapes of membership functions

The expected value of fuzzy variable is defined by Liu and Liu [2] as (6):

$$
E[\xi]=\int_{0}^{\infty} C r\{\xi \geq x\} d x-\int_{-\infty}^{0} \operatorname{Cr}\{\xi \leq x\} d x
$$

provided that the two integrals are finite.

Furthermore, the variance is defined in [2] as (7) and semivariance in [5] as (8).

$$
\begin{gathered}
V[\xi]=E\left[(\xi-E[\xi])^{2}\right] \\
S V[\xi]=E\left[\left[(\xi-E[\xi])^{-}\right]^{2}\right]
\end{gathered}
$$

where

$$
(\xi-E[\xi])^{-}=\left\{\begin{array}{cc}
\xi-E[\xi], & \text { for } \xi \leq E[\xi] \\
0, & \text { for } \xi>E[\xi]
\end{array}\right.
$$

Note that in the case of symmetric membership function, variance equals semivariance. A general case $V[\xi] \geq S V[\xi]$, because it includes also the above-expected value deviation.

The entropy measure was presented by $\mathrm{Li}$ and Liu [14] as (10).

$$
H[\xi]=\int_{-\infty}^{\infty} S(C r(\xi=x)) d x
$$

where $S(t)=-t \ln (t)-(1-t) \ln (1-t)$
The last considered risk measure is value-at-risk. The VaR of an investment is the credibility of the greatest loss at some confidence level. Wang, Watada and Pedrycz [16] defined the credibilistic VaR as (11).

$$
\operatorname{VaR}(\alpha)=\sup \{x \mid \operatorname{Cr}(\eta \geq x) \geq \alpha\}
$$

for a risk confidence level $1-\alpha$. In this paper we consider fuzzy variable for return rate. Therefore credibilistic VaR quantifies the minimum amount $x$, which the fuzzy return rate may take with a credibility of no less than $\alpha$ (12).

$$
\operatorname{VaR}(\alpha)=\inf \{x \mid \operatorname{Cr}(\xi \leq x) \geq \alpha\}
$$

Definition (12) in the case of continuous functions is equivalent to (13).

$$
\operatorname{VaR}(\alpha)=\sup \{x \mid \operatorname{Cr}(\xi \leq x) \leq \alpha\}
$$

Value at risk is defined by (13) and is interpreted as follows: $\operatorname{VaR}(\alpha)=y$ means that there is at most $\alpha$ percent credibility that the value of fuzzy rate of return falls below $y$. Commonly used confidence levels are $99 \%$ and $95 \%$.

In brief Table 1 present the above-mentioned measures for the considered shapes.

\subsection{Portfolio selection problems}

Modern portfolio theory attempts to maximize portfolio expected return for a given amount of portfolio risk, or equivalently minimize the risk for a given level of expected return, by carefully choosing the proportions of various assets.

Let: $\xi=\left(\xi_{1}, \xi_{2}, \ldots, \xi_{n}\right)$ be a vector of fuzzy return assets $1,2, \ldots, n, x=\left(x_{1}, x_{2}, \ldots, x_{n}\right)$ be a vector of shares in the portfolio, where $x_{i}$ - the share of $i$-th assets in the portfolio. We assume also two standard constraints for all task:

- that there is no short sale, so $\forall_{i} x_{i} \geq 0$

$-\sum_{i=1}^{n} x_{i}=1$.

In this analysis the expected value of fuzzy return is taken into consideration as measure of gain and uncertainty of portfolio is presented by variance (7), semivariance (8), entropy (10) or value-at-risk (12). Comprehensive overview of fuzzy portfolio selection models can be found in [7]. In survey we consider following tasks: 
Table 1. Basic measures for different shapes

\begin{tabular}{|c|c|c|c|c|}
\hline Shape & 1 & 2 & 3 & 4 \\
\hline$E$ & $\frac{a+2 b+c}{4}$ & $\frac{a+b+c}{3}$ & $\frac{a+4 b+c}{6}$ & $\frac{a+2 b+c}{4}$ \\
\hline$S V=V^{*}$ & $\frac{(b-a)^{2}}{6}$ & $\frac{(b-a)^{2}}{4}$ & $\frac{(b-a)^{2}}{12}$ & $\frac{(b-a)^{2}}{6}$ \\
\hline$H$ & $\frac{c-a}{2}$ & $(c-a) \frac{\frac{\pi}{2}-\ln 2}{3}$ & $\begin{array}{c}(c-a) \\
\left(\frac{4}{3}+\ln 2+\frac{(4 \sqrt{2})}{3} \ln (\sqrt{2}-1)\right)\end{array}$ & $\left(\frac{4}{3}+2 \ln 2-\frac{17}{12} \ln 3+\frac{\sqrt{2} \ln (\sqrt{2}-1)}{3}-\frac{\sqrt{2}}{3} \tan ^{-1}\left(\frac{\sqrt{2}}{2}\right)\right)$ \\
\hline$V a R^{* *}$ & $2 \alpha(b-a)+$ & $1-2 \alpha(b-a)+b$ & $\sqrt{2 \alpha}(b-a)+a$ & $\sqrt{\alpha}(b-a)+a$ \\
\hline
\end{tabular}

$*$ for symmetric membership functions

** assuming that $\alpha \leq 0.25$

1. credibilistic mean-variance [3]

1.1 the task of maximization expected value

$$
\begin{gathered}
\max E\left[\theta \in \Theta: \sum x_{i} \xi_{i}(\theta)\right] \\
\text { s.t.: } V\left[\theta \in \Theta: \sum x_{i} \xi_{i}(\theta)\right] \leq \mathrm{v},
\end{gathered}
$$

where $v$ specified maximum acceptable level of variance.

2.1 the task of minimizing the variance

$$
\begin{gathered}
\min V\left[\theta \in \Theta: \sum x_{i} \xi_{i}(\theta)\right], \\
\text { s.t.: } E\left[\theta \in \Theta: \sum x_{i} \xi_{i}(\theta)\right] \geq \eta
\end{gathered}
$$

where $\lambda$ specified expected return rate.

2. mean-semivariance [5]

1.2 the task of maximization expected value

$$
\begin{gathered}
\max E\left[\theta \in \Theta: \sum x_{i} \xi_{i}(\theta)\right] \\
\text { s.t.:SV }\left[\theta \in \Theta: \sum x_{i} \xi_{i}(\theta)\right] \leq \mathrm{v},
\end{gathered}
$$

where $v$ specified maximum acceptable level of semivariance.

2.2 the task of minimizing the semivariance

$$
\begin{aligned}
& \min S V\left[\theta \in \Theta: \sum x_{i} \xi_{i}(\theta)\right], \\
& \text { s.t.: } E\left[\theta \in \Theta: \sum x_{i} \xi_{i}(\theta)\right] \geq \eta
\end{aligned}
$$

3. mean-entropy [6]

1.3 the task of maximization expected value

$$
\begin{gathered}
\max E\left[\theta \in \Theta: \sum x_{i} \xi_{i}(\theta)\right] \\
\text { s.t.: } H\left[\theta \in \Theta: \sum x_{i} \xi_{i}(\theta)\right] \leq \mathrm{v},
\end{gathered}
$$

where $v$ specified maximum acceptable level of entropy.

2.3 the task of minimizing the entropy

$$
\begin{gathered}
\min H\left[\theta \in \Theta: \sum x_{i} \xi_{i}(\theta)\right], \\
\text { s.t.: } E\left[\theta \in \Theta: \sum x_{i} \xi_{i}(\theta)\right] \geq \eta
\end{gathered}
$$

\section{4. mean-VaR [17]}

1.4 the task of maximization expected value

$$
\begin{gathered}
\max E\left[\theta \in \Theta: \sum x_{i} \xi_{i}(\theta)\right] \\
\text { s.t.:VaR }\left[\theta \in \Theta: \sum x_{i} \xi_{i}(\theta)\right] \leq \mathrm{v},
\end{gathered}
$$

where $v$ specified maximum acceptable level of value-at-risk.

2.4 the task of minimizing value-at-risk

$$
\begin{aligned}
& \min \operatorname{VaR}\left[\theta \in \Theta: \sum x_{i} \xi_{i}(\theta)\right], \\
& \text { s.t.: } E\left[\theta \in \Theta: \sum x_{i} \xi_{i}(\theta)\right] \geq \eta
\end{aligned}
$$

If the considered objective is to maximize the investment return for an appointed level of risk - the tasks are pointed as $1 . *$, where * - task number with particular uncertain measure, or to minimize the investment risk for a given level of return - the tasks pointed as $2 . *$.

\section{Research}

The aim of the study is to determine whether the triangular membership function is sufficient to present a variety of fuzzy returns. So two auxiliary hypotheses were examined:

1. Optimization result is independent of the shape of the membership function

2. There are no statistically significant differences between results of optimization with triangular fuzzy return rate and with 2, 3, 4 shape fuzzy return rate. 
The first hypothesis is checked analytically and the second one empirically.

Due to the difficulties in analytical calculations of some measurements of uncertainty in the general case, at first the special case - the symmetric fuzzy returns are examined. If the results of the special symmetric case (labeled 's' next to the task number) do not differ from each other, then the test will be generalized to the asymmetric case (labeled with 'ns'). Optimization tasks are solved in two variants. First, assuming the same parameters $a, b$, $c$ of different shapes (see Fig. 1) - labeled variant (a), and the other assuming the same value of parameter $b$ and area under the graph of membership function (see Fig. 1) - labeled variant (b). Let $a_{i}, b_{i}, c_{i}$ - be parameter $a$ for $i$-th membership function's shape, then in variant (b) the following formulas occur: $\left(c_{2}-a_{2}\right)=\frac{3}{4}\left(c_{1}-a_{1}\right),\left(c_{3}-a_{3}\right)=$ $\frac{3}{2}\left(c_{1}-a_{1}\right),\left(c_{3}-a_{3}\right)=\frac{3}{2}\left(c_{1}-a_{1}\right)$. This implies that expected value of fuzzy variable is independent of the shape and is equal: $E[\xi]=\frac{a_{1}+2 b_{1}+c_{1}}{4}$.

\subsection{Analytical study}

The tasks 1.3, 1.4, 2.3, 2.4 are linear programming problems. The basic property of the linear programming solutions is that if there is an optimal solution, then it is one of the base solutions.

The tasks 1.1, 1.2, 2.1, 2.2 are quadratic programming problems. Under differentiability, convexity and constraint qualifications, the Karush-Kuhn-Tucker (KKT) conditions provide necessary and sufficient conditions for a solution to be optimal. Tasks with symmetric membership function satisfy the regularity constraints for KKT. In the case of maximization tasks utilizing symmetrical rates of return, all tasks, regardless of the type and shape have the same objective function and differ in terms of the constraint function. While in the case of minimization, all tasks have a constraint on the minimum expected value expressed by uncertainty: $b \geq \eta$. Tasks differ with the objective function. It allows, in the symmetrical case for assumption regarding fixed parameters (variant $(a)$ ) and fixed fields under the graph (variant $(b)$ ), to determine the following form of the tasks' optimal solutions:

\section{$1.1,1.2$}

$$
\begin{gathered}
x_{k}=\frac{\left(b_{j}-a_{j}\right)-\sqrt{\frac{v}{C}}}{\left(b_{j}-a_{j}\right)-\left(b_{k}-a_{k}\right)}-\sum_{i=1}^{n} \frac{\left(b_{j}-a_{j}\right)-\left(b_{i}-a_{i}\right)}{\left(b_{j}-a_{j}\right)-\left(b_{k}-a_{k}\right)} t_{i}, \\
x_{j}=\frac{\sqrt{\frac{v}{C}}-\left(b_{k}-a_{k}\right)}{\left(b_{j}-a_{j}\right)-\left(b_{k}-a_{k}\right)}-\sum_{i=1}^{n} \frac{\left(b_{i}-a_{i}\right)-\left(b_{j}-a_{j}\right)}{\left(b_{j}-a_{j}\right)-\left(b_{k}-a_{k}\right)} t_{i}, \\
\forall i \neq j, k=x_{i} \geq 0, \\
t_{j}=t_{k}=0 \\
\Leftrightarrow \quad\left(\left(b_{j}>b_{k} \wedge\left(\left(b_{k}-a_{k}\right) \geq\left(b_{j}-a_{j}\right)\right) \wedge\right.\right. \\
\left.\left(\left(b_{j}-a_{j}\right) b_{k} \geq\left(b_{k}-a_{k}\right) b_{j}\right)\right) \vee \\
\left(b_{j}<b_{k} \wedge\left(\left(b_{k}-a_{k}\right) \leq\left(b_{j}-a_{j}\right)\right) \wedge\right. \\
\left.\left(\left(b_{j}-a_{j}\right) b_{k} \leq\left(b_{k}-a_{k}\right) b_{j}\right)\right) \wedge \\
\forall b_{i} \geq \frac{\left(b_{i}-a_{i}\right)\left(b_{j}-b_{k}\right)-b_{k}\left(b_{j}-a_{j}\right)+b_{j}\left(b_{k}-a_{k}\right)}{C\left(\left(b_{j}-a_{j}\right)-\left(b_{k}-a_{k}\right)\right) .}
\end{gathered}
$$

\section{3}

$x^{B}=\left[\begin{array}{ll}x_{k} & x_{j}\end{array}\right]=\left[\begin{array}{ll}\Leftrightarrow \frac{\left(b_{j}-a_{j}\right)-\frac{v}{C}}{\left(b_{j}-a_{j}\right)-\left(b_{k}-a_{k}\right)} & \frac{\frac{v}{C}-\left(b_{k}-a_{k}\right)}{\left(b_{j}-a_{j}\right)-\left(b_{k}-a_{k}\right)}\end{array}\right]$
$\left(b_{j}-a_{j}<b_{k}-a_{k}\right) \wedge\left(b_{j} \leq b_{k}\right)$,
$\forall_{i} b_{i} \leq \frac{(24)}{\left(b_{i}-a_{i}\right)\left(b_{j}-b_{k}\right)-b_{j}\left(b_{k}-a_{k}\right)+b_{k}\left(b_{j}-a_{j}\right)}$
$C\left(\left(b_{j}-a_{j}\right)-\left(b_{k}-a_{k}\right)\right)$

$$
\left(b_{j}-a_{j} \geq b_{k}-a_{k}\right) \wedge\left(b_{j}>b_{k}\right) .
$$

1.4

for shape 1, 3 and 4 :

$$
\begin{gathered}
x_{k}=\frac{C_{3} a_{j}-C_{1}\left(C_{2} a_{j}-b_{j}\right)+v}{C_{1}\left(b_{j}-C_{2} a_{j}\right)+C_{3} a_{j}-C_{1}\left(b_{k}-C_{2} a_{k}\right)-C_{3} a_{k}} \\
x_{j}=\frac{-v-C_{3} a_{k}-C_{1}\left(b_{k}-C_{2} a_{k}\right)}{C_{1}\left(b_{j}-C_{2} a_{j}\right)+C_{3} a_{j}-C_{1}\left(b_{k}-C_{2} a_{k}\right)-C_{3} a_{k}}, \\
\forall_{i} b_{i} \geq \frac{\left(b_{j}-b_{k}\right)\left(C_{1}\left(C_{2} a_{i}-b_{i}\right)-C_{3} a_{i}\right)+b_{j}\left(C_{1}\left(C_{2} a_{k}-b_{k}\right)-C_{3} a_{k}\right)}{C_{1}\left(C_{j}-C_{2} a_{j}\right)+C_{3} a_{j}-C_{1}\left(b_{k}-C_{2} a_{k}\right)-C_{3} a_{k}} \\
\quad+\frac{\left.b_{k}\left(C_{3}\right)-C_{1}\left(C_{2} a_{j}-b_{j}\right)\right)}{C_{1}\left(b_{j}-C_{2} a_{j}\right)+C_{3} a_{j}-C_{1}\left(b_{k}-C_{2} a_{k}\right)-C_{3} a_{k}},
\end{gathered}
$$

where

$$
\begin{gathered}
C_{1}=\left\{\begin{array}{cc}
2 \alpha & \text { for shape } 1(\mathrm{a}),(\mathrm{b}), \\
\sqrt{2 \alpha} & \text { for shape } 3(\mathrm{a}),(\mathrm{b}), \\
\sqrt{\alpha} & \text { for shape } 4(\mathrm{a}),(\mathrm{b}),
\end{array}\right. \\
C_{2}=\left\{\begin{array}{lc}
\frac{3}{2} & \text { for shape } 3(\mathrm{~b}), \\
1 & \text { otherwise }
\end{array}\right. \\
C_{3}=\left\{\begin{array}{cc}
\frac{3}{2} & \text { for shape } 3(\mathrm{~b}), \\
1 & \text { otherwise }
\end{array}\right.
\end{gathered}
$$

for shape 2:

$$
\begin{aligned}
x_{k} & =\frac{b_{j}-C_{1}\left(C_{2} a_{j}-b_{j}\right)+v}{C_{1}\left(b_{j}-C_{2} a_{j}\right)+b_{j}-C_{1}\left(b_{k}-C_{2} a_{k}\right)-b_{k}}, \\
x_{j} & =\frac{-v-b_{k}-C_{1}\left(b_{k}-C_{2} a_{k}\right)}{C_{1}\left(b_{j}-C_{2} a_{j}\right)+b_{j}-C_{1}\left(b_{k}-C_{2} a_{k}\right)-b_{k}},
\end{aligned}
$$




$$
\begin{gathered}
\forall_{i} b_{i} \geq\left(b_{j}-b_{k}\right)\left(\left(C_{1}\left(C_{2} a_{i}-b_{i}\right)-b_{i}\right)+\right. \\
b_{j}\left(C_{1}\left(C_{2} a_{k}-b_{k}\right)-b_{k}\right)+ \\
\left.b_{k}\left(b_{j}-C_{1}\left(C_{2} a_{j}-b_{j}\right)\right)\right) /\left(C_{1}\left(b_{j}-C_{2} a_{j}\right)+\right. \\
\left.b_{j}-C_{1}\left(b_{k}-C_{2} a_{k}\right)-b_{k}\right)
\end{gathered}
$$

where

$$
\begin{aligned}
& C_{1}=\{\sqrt{1-2 \alpha} \text { for variant (a),(b) } \\
& C_{2}=\left\{\begin{array}{cc}
\frac{3}{4} & \text { for variant (b) } \\
1 & \text { otherwise }
\end{array}\right.
\end{aligned}
$$

\section{$2.1,2.2$}

$$
\begin{gathered}
x_{k}=\frac{b_{j}-\eta}{b_{j}-b_{k}}-\sum_{i=1}^{n} \frac{b_{j}-b_{i}}{b_{j}-b_{k}} t_{i}, \\
x_{j}=\frac{\eta-b_{k}}{b_{j}-b_{k}}-\sum_{i=1}^{n} \frac{b_{i}-b_{k}}{b_{j}-b_{k}} t_{i}, \\
\forall_{i \neq j, k} x_{i}=t_{i}, \\
t_{j}=t_{k}=0 \\
\Leftrightarrow\left(b_{j}>b_{k} \wedge\left(\left(b_{j}-a_{j}\right) \geq\left(b_{k}-a_{k}\right)\right)\right. \\
\left.\wedge\left(\left(b_{j}-a_{j}\right) b_{k} \geq\left(b_{k}-a_{k}\right) b_{j}\right)\right) \vee \\
\left(b_{j}<b_{k} \wedge\left(\left(b_{j}-a_{j}\right)<\left(b_{k}-a_{k}\right)\right)\right. \\
\left.\wedge\left(\left(b_{j}-a_{j}\right) b_{k}>\left(b_{k}-a_{k}\right) b_{j}\right)\right) \wedge \\
\forall_{i} b_{i} \geq \frac{\left(b_{i}-a_{i}\right)\left(b_{k}-b_{j}\right)-b_{j}\left(b_{k}-a_{k}\right)+b_{k}\left(b_{j}-a_{j}\right)}{\left(b_{j}-a_{j}\right)-\left(b_{k}-a_{k}\right)}
\end{gathered}
$$

\section{3}

$$
\begin{gathered}
x_{j}=\frac{b_{j}-\eta}{b_{j}-b_{k}}, \\
x_{k}=\frac{\eta-b_{k}}{b_{j}-b_{k}}, \\
\Leftrightarrow\left(\left(b_{j}-a_{j}\right) \leq\left(b_{k}-a_{k}\right) \wedge\left(b_{j}<b_{k}\right)\right) \\
\vee\left(\left(b_{j}-a_{j}\right) \geq\left(b_{k}-a_{k}\right) \wedge\left(b_{j}>b_{k}\right)\right) \wedge \\
\forall_{i} b_{i} \geq \frac{\left(b_{i}-a_{i}\right)\left(b_{k}-b_{j}\right)-b_{j}\left(b_{k}-a_{k}\right)+b_{k}\left(b_{j}-a_{j}\right)}{\left(b_{j}-a_{j}\right)-\left(b_{k}-a_{k}\right)}
\end{gathered}
$$

2.4 for shape $1,3,4$ :

$$
\begin{gathered}
x_{j}=\frac{\eta-b_{k}}{b_{j}-b_{k}}, \\
x_{k}=\frac{b_{j}-\eta}{b_{j}-b_{k}}, \\
\Leftrightarrow \forall_{i} C_{1}\left(C_{2} a_{i}-b_{i}\right)-a_{i} \geq \\
+\frac{b_{j}\left(C_{1}\left(C_{2} b_{k}-b_{k}\right)-a_{k}\right)}{b_{j}-b_{k}},
\end{gathered}
$$$$
\frac{b_{i}\left(C_{1}\left(C_{2} a_{j}-b_{j}\right)-a_{j}-C_{1}\left(C_{2} a_{k}-b_{k}\right)+a_{k}\right)-b_{k}\left(C_{1}\left(C_{2} a_{j}-b_{j}\right)-a_{j}\right)}{b_{j}-b_{k}}
$$

where

$$
\begin{gathered}
C_{1}=\left\{\begin{array}{cc}
2 \alpha & \text { for shape } 1(\mathrm{a}),(\mathrm{b}) \\
\sqrt{2 \alpha} & \text { for shape } 3(\mathrm{a}),(\mathrm{b}) \\
\sqrt{\alpha} & \text { for shape 4 (a), (b) }
\end{array}\right. \\
C_{2}=\left\{\begin{array}{cc}
\frac{3}{2} & \text { for shape } 3(\mathrm{~b}), \\
1 & \text { otherwise }
\end{array}\right.
\end{gathered}
$$

for shape 2:

$$
\begin{gathered}
x_{j}=\frac{\eta-b_{k}}{b_{j}-b_{k}}, \\
x_{k}=\frac{b_{j}-\eta}{b_{j}-b_{k}}, \\
\Leftrightarrow \frac{b_{i}\left(C_{1}\left(C_{2} a_{j}-b_{j}\right)-b_{j}-C_{1}\left(C_{2} a_{k}-b_{k}\right)+b_{k}\right)-b_{k}\left(C_{1}\left(C_{2} a_{j}-b_{j}\right)-b_{j}\right)}{b_{j}-b_{k}} \\
+\frac{b_{j}\left(C_{1}\left(C_{2} a_{k}-b_{k}\right)-b_{k}\right)}{b_{j}-b_{k}}
\end{gathered}
$$

where

$$
\begin{gathered}
C_{1}=\{\sqrt{1-2 \alpha} \text { for variant (a),(b) } \\
C_{2}=\left\{\begin{array}{cc}
\frac{3}{4} & \text { for variant (b), } \\
1 & \text { otherwise }
\end{array}\right.
\end{gathered}
$$

The following conclusions can be drawn from the analysis: The optimal solution of the expected value maximization task at a given maximum value of entropy, variance, semivariance, value at risk and conditional value at risk for symmetrical numbers does not depend on the adapted shape of the fuzzy variable. The optimal solution of the entropy minimization task at a given expected value for symmetrical numbers does not depend on the adapted shape of the fuzzy variable. The optimal solution of the variance minimization task at a given expected value for symmetrical numbers does not depend on the adapted shape of the fuzzy variable. With a given shape, the solutions of the entropy minimization and variance (semivariance) minimization tasks at a predetermined minimum expected value are equal, under the condition: $\forall_{i} b_{i}>\frac{\left(\left(b_{i}-a_{i}\right)\left(b_{k}-b_{j}\right)-b_{j}\left(b_{k}-a_{k}\right)+b_{k}\left(b_{j}-a_{j}\right)\right)}{\left.\left(\left(b_{j}-a_{j}\right)-\left(b_{k}-a_{k}\right)\right)\right)}$.

\subsection{Numerical simulation}

If the analytical verification showed that the optimal portfolio is dependent on the shape of a membership function, a simulation was performed to examine the differences between the portfolio obtained with different shapes. In order to examine differences between selected portfolios according to the membership function shape the following simulation steps have been used:

1. 10 draws of 3 random (or 2 for symmetric case) parameters defining membership function $\mu_{\xi_{i}}(a, b, c)$,

2. calculation tasks, every task with every shape of membership function, 
3. calculation differences between optimal share vectors (between results of tasks using shapes 1 and 2,1 and 3,1 and 4),

4. 5000 repetitions of steps $1-3$,

5. calculation descriptive statistics,

6. The Wilcoxon signed-ranks test.

After the first step we have simulated value of fuzzy variable for a collection of 10 assets, from which we will select portfolios. Step 2 is to solve tasks (14)(21) separately for each shape. The solutions are calculated assuming the minimum expected value is not less than the average expected value randomly selected variables ( $\eta$ value) and the maximum uncertainty measure is not more than the average value of uncertain measures form selected variables $(v$ value). Averages value was selected for coefficients to provide non-empty set of feasible solutions for each task. In step 3 - for calculating differences a different coefficient (1-cosine similarity) is used, so 0 means exactly the same result, 1 indicates a completely different portfolio.

Beside descriptive statistics of different coefficient, the Wilcoxon Signed-Rank Test is used to evaluate of the hypothesis. The Wilcoxon Signed-Rank Test is the nonparametric test equivalent to the paired Student's t-test, t-test for matched pairs, or the t-test for dependent samples, when the population cannot be assumed to be normally distributed. The null hypothesis in this test is that median difference between the pairs is zero. More information about the test can be found in [15]. In considered case the data collected with the task $(1.1-1.4,2.1-2.5)$ for linear shape functions is tested respectively to the same task result for the shapes: 2, 3 and 4, in order to determine whether there was a statistically significant change.

\section{Results}

As shown in Table 1 variance (semivariance), entropy for particular shapes differs only in fixed, so obviously have no effect on the result of minimizing the function, which has been proven analytically. The results of analytical study concerning symmetric fuzzy numbers has shown that all the tasks to maximize the expected value of fuzzy rate of return and the task of minimizing the value at risk are dependent on the shape of the membership function. Analyzing the task of minimizing entropy with asymmetric fuzzy numbers, the solution is dependent on the shape in variant (a) and independent in (b).

Table 2. Statistics of different coefficients

\begin{tabular}{|c|c|c|c|c|c|c|}
\hline \multirow{2}{*}{\begin{tabular}{|l} 
\\
Task \\
\end{tabular}} & \multirow{2}{*}{\begin{tabular}{|l} 
Shape \\
Variant
\end{tabular}} & \multicolumn{2}{|c|}{ I-II } & \multicolumn{2}{|c|}{ I-III } & \multirow{2}{*}{\begin{tabular}{|l} 
I-IV \\
(a)/(b)
\end{tabular}} \\
\hline & & (a) & (b) & (a) & (b) & \\
\hline \multirow{6}{*}{$1.2 \mathrm{~s} *$} & $\min$ & 0 & 0 & 0 & 0 & 0 \\
\hline & Q1 & 0,0190 & $6,473 \mathrm{e}-13$ & $4,465 \mathrm{e}-13$ & 0,0015 & 0 \\
\hline & median & 0,0324 & 0,0062 & 0,0900 & 0,0030 & 0 \\
\hline & mean & 0,0560 & 0,0114 & 0,1153 & 0,0062 & 0 \\
\hline & Q3 & 0,0629 & 0,0117 & 0,1731 & 0,0058 & 0 \\
\hline & $\max$ & 0,5406 & 0,4949 & 0,6874 & 0,4823 & 0 \\
\hline \multirow{6}{*}{$1.3 \mathrm{~s}$} & $\min$ & 0 & 0 & 0 & 0 & 0 \\
\hline & Q1 & 0,0872 & 0,2010 & 0,2883 & 0,1128 & 0,0027 \\
\hline & median & 0,1774 & 0,3569 & 0,4381 & 0,2181 & 0,0057 \\
\hline & mean & 0,2135 & 0,3628 & 0,4545 & 0,2503 & 0,0136 \\
\hline & Q3 & 0,3186 & 0,4881 & 0,6070 & 0,3785 & 0,0121 \\
\hline & $\max$ & 0,9960 & 1 & 1 & 0,9983 & 0,4318 \\
\hline \multirow{6}{*}{$1.3 \mathrm{~ns}$} & $\min$ & 0 & 0 & 0 & 0 & 0 \\
\hline & Q1 & 0,1197 & 0,2013 & 0,2921 & 0,1569 & 0,0048 \\
\hline & median & 0,2916 & 0,4307 & 0,4655 & 0,3392 & 0,0102 \\
\hline & mean & 0,2875 & 0,4143 & 0,4904 & 0,3220 & 0,0264 \\
\hline & Q3 & 0,4308 & 0,5746 & 0,7447 & 0,4592 & 0,0222 \\
\hline & $\max$ & 0,9924 & 1 & 1 & 0,9931 & 0,4922 \\
\hline \multirow{6}{*}{$1.4 \mathrm{~s}$} & $\min$ & 0,1599 & 0,1406 & 0 & 0 & 0 \\
\hline & Q1 & 0,7841 & 0,7373 & 0 & 0 & 0 \\
\hline & median & 1 & 1 & 0 & 0 & 0 \\
\hline & mean & 0,8757 & 0,8591 & 0,0272 & 0,0043 & 0,0080 \\
\hline & Q3 & 1 & 1 & 0,0203 & 0 & 0,0021 \\
\hline & $\max$ & 1 & 1 & 0,5515 & 0,3752 & 0,4919 \\
\hline \multirow{6}{*}{$1.4 \mathrm{~ns}$} & $\min$. & 0,0187 & 0,0177 & 0 & 0 & 0 \\
\hline & Q1 & 0,7047 & 0,6679 & 0,0040 & 0 & 0 \\
\hline & median & 0,9311 & 0,8992 & 0,0636 & 0,0020 & 0,0094 \\
\hline & mean & 0,8343 & 0,8158 & 0,1332 & 0,0190 & 0,0317 \\
\hline & Q3 & 1 & 1 & 0,1880 & 0,0166 & 0,0319 \\
\hline & $\max$ & 1 & 1 & 1 & 0,4973 & 0,6877 \\
\hline \multirow{6}{*}{$2.4 \mathrm{~s}$} & $\min$ & 0,4402 & 0,4402 & 0 & 0 & 0 \\
\hline & Q1 & 1 & 1 & 0 & 0 & 0 \\
\hline & median & 1 & 1 & 0 & 0 & 0 \\
\hline & mean & 0,9871 & 0,9870 & 0,0153 & 0,0250 & 0,0120 \\
\hline & Q3 & 1 & 1 & 0 & 0 & 0 \\
\hline & $\max$ & 1 & 1 & 0,5673 & 0,9031 & 0,5673 \\
\hline \multirow{6}{*}{$2.4 \mathrm{~ns}$} & $\min$ & 0,0020 & 0 & 0 & 0 & 0 \\
\hline & Q1 & 0,8469 & 0,8852 & 0,0020 & 0 & 0 \\
\hline & median & 1 & 1 & 0,0106 & 0 & 0 \\
\hline & mean & 0,8890 & 0,9016 & 0,0584 & 0,0553 & 0,0546 \\
\hline & Q3 & 1 & 1 & 0,0455 & 0,0013 & 0,0022 \\
\hline & $\max$ & 1 & 1 & 0,9865 & 0,8795 & 1 \\
\hline
\end{tabular}

$* s$ for symmetric membership functions ( $n s$ non-symmetric) 
Table 3. P-values of Wilcoxon signed-rank test

\begin{tabular}{|c|c|c|c|c|c|}
\hline \multirow{2}{*}{ Task } & \multicolumn{2}{|c|}{ shapes: 1 and 2} & \multicolumn{2}{c|}{ shapes: 1 and 3} & shapes: 1 and 4 \\
\cline { 2 - 6 } & (a) & (b) & (a) & (b) & (a)/(b) \\
\hline $1.2 \mathrm{~s} *$ & 0,4807 & 0,3137 & 0,0265 & 0,4952 & 1 \\
\hline $1.3 \mathrm{~s}$ & 0,2778 & 0,0153 & $9,80 \mathrm{e}-05$ & 0,1610 & 0,9509 \\
\hline $1.3 \mathrm{~ns}$ & $1,08 \mathrm{e}-05$ & $2,47 \mathrm{e}-12$ & $2,54 \mathrm{e}-17$ & $7,62 \mathrm{e}-06$ & 0,7225 \\
\hline $1.4 \mathrm{~s}$ & $3,63 \mathrm{e}-58$ & $3,63 \mathrm{e}-58$ & 0,0056 & 0,3299 & 0,1409 \\
\hline $1.4 \mathrm{~ns}$ & $3,70 \mathrm{e}-21$ & $3,70 \mathrm{e}-21$ & 0,0025 & 0,0639 & 0,0307 \\
\hline $2.3 \mathrm{~ns}$ & 0,9826 & - & 0,9895 & - & 0,9999 \\
\hline $2.4 \mathrm{~s}$ & 0,4112 & 0,3805 & 0,9613 & $2,06 \mathrm{e}-29$ & 0,7111 \\
\hline $2.4 \mathrm{~ns}$ & 0,0658 & 0,5544 & 0,0888 & $1,86 \mathrm{e}-43$ & 0,8978 \\
\hline
\end{tabular}

$* s$ for symmetric membership functions ( $n s$ non-symmetric)

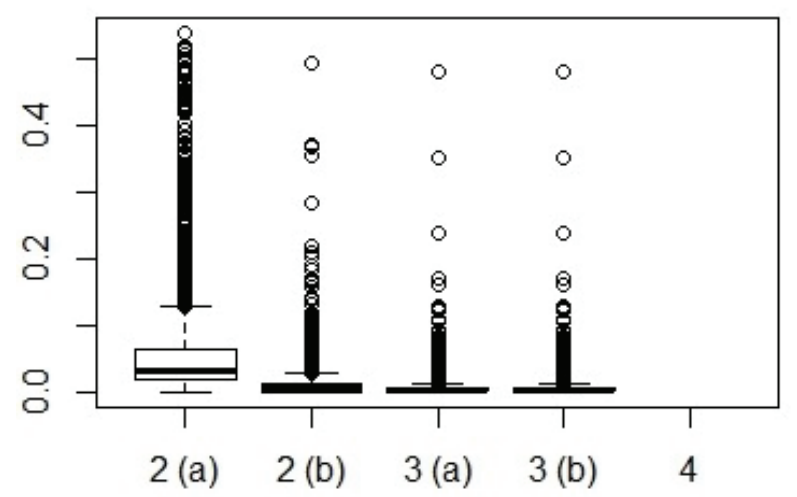

Figure 2. Differences - task 1.2 for symmetric numbers

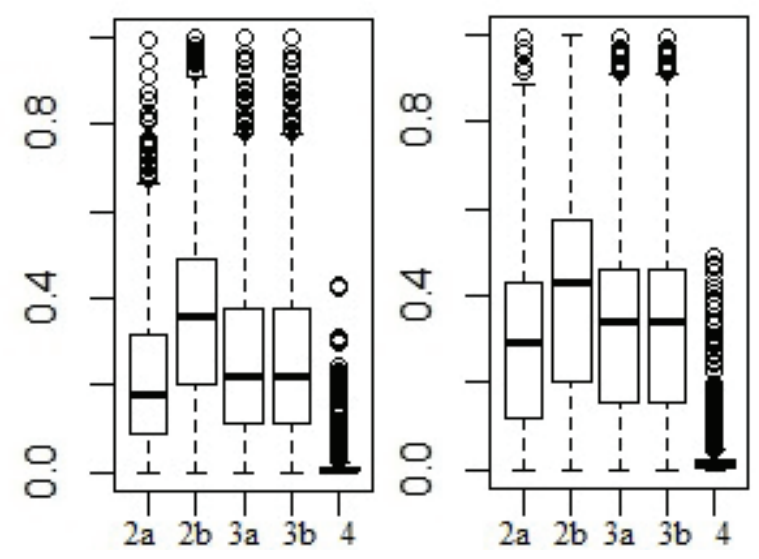

Figure 3. Differences - task 1.3

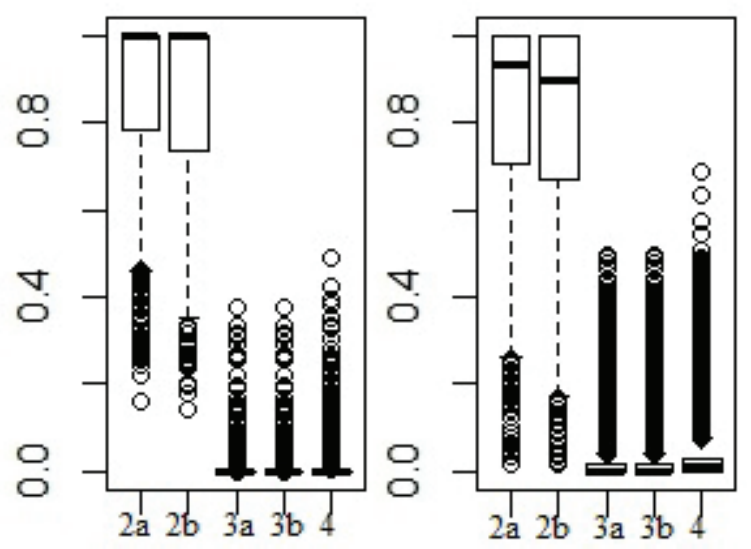

Figure 4. Differences - task 1.4

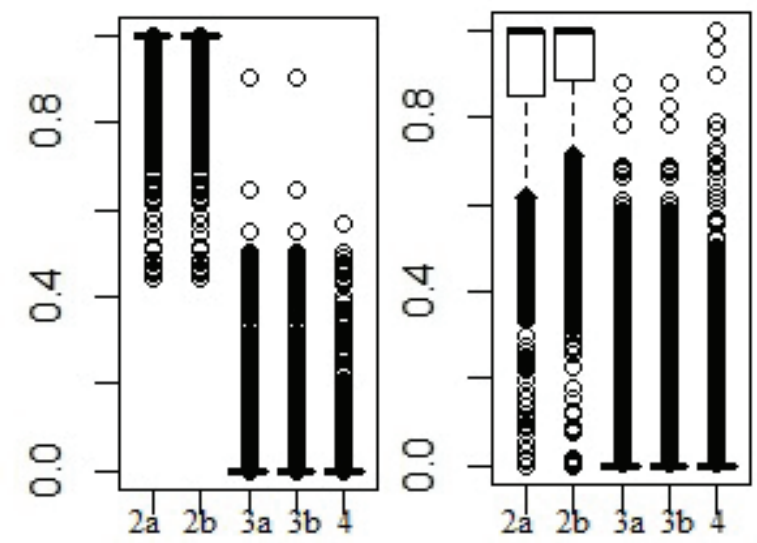

Figure 5. Differences - task 2.4

The tasks: 1.1, 1.2, 1.3, 1.4, 2.1, 2.2, 2.3 (only variant (a)), 2.4 were studied for second hypotheses by simulation. The descriptive statistics of differences of solution vectors are included in Table 2. Boxplots for difference coefficient between vectors solutions shown in Fig. 2 - 5. On the graph $\mathrm{x}$-axis presents the different shapes for variants (a) and (b), $y$-axis values of coefficient from 0 to 1 . Graphs on the left side show symmetric cases of fuzzy variable (on the right side non-symmetric). P-values of Wilcoxon signed-rank test are shown in Table 3. Taking into account the 0,05 significance level the null hypothesis should be rejected for shape 1 and 2 for task $1.3 \mathrm{~ns}^{2}, 1.4 \mathrm{~s}, 1.4 \mathrm{~ns}$, and for shape 1 and 3 for $1.3 \mathrm{~s}(\mathrm{a}), 1.3 \mathrm{~ns}, 1.4 \mathrm{~s}, 1.4 \mathrm{~ns}, 2.4 \mathrm{~s}(\mathrm{~b}), 2.4 \mathrm{~ns}(\mathrm{~b})$. In short we can say that for tasks using asymmetric fuzzy numbers change the membership functions shapes from linear to 2 and 3 shape has a signifi-

\footnotetext{
${ }^{2} s$ is for symmetric membership functions, $n s$ non-symmetric
} 
cant influence on result of optimization. The statistics show that regardless of task number the lowest differences are between result for shapes 1 and 4 (Table 2). The biggest median of differences in this case is only 0,0546 . Furthermore, irrespective of the task, variant and selected samples there is no reason to reject the null hypothesis of the Wilcoxon test for 4 shapes.

For shapes 1 and 2, the result differences are the smallest for task 1.2s (median: 0,0324 (a), 0,0062 (b)) but tasks consider VaR as risk measure have completely different output (f.e. median 1 for $1.4 \mathrm{~s}$, $0,9311$ for $1.4 \mathrm{~ns}(\mathrm{a}))$, for this task also p-value is low, so null hypothesis should be rejected.

Comparing shapes 1 and 3 the median for different tasks is in the range from $0(1.4 \mathrm{~s})$ to $0,4381(1.3 \mathrm{~s}$ (a)). Moreover in variant $b$ the differences are always clearly smaller.

When considering the results, the differences are clearly dependent on the adopted measure of uncertainty. Models using SV and V are more resistant to changes in shape of the membership function for the task using the task of $\mathrm{H}$. A VaR behaves unexpectedly and has major differences when comparing shapes 1 and 2, and small for 1 and 3, which cannot be seen in other tasks.

\section{Conclusion}

The aim of this article was to determine whether the triangular membership function is sufficient to present a variety of fuzzy returns. In general, the shape of the membership function choice of fuzzy returns has an impact on the result of optimization. However, some uncertain measures are more resistant to shape changes, which may also be significant selection criterion for optimization models. Simulation and analysis of optimization tasks confirmed that the number of triangular can be a good approximation of the shape of 4 , but in the case of shape 2 and 3, the results are no longer so obvious. Thus, in contrast to research carried out in area of fuzzy controllers, in the case of portfolio optimization shape change from linear to Gaussian does not improve results, cause no impact, but significantly increases the computational complexity. An interesting finding is a strong dependence of resistance to change shape depending on the measure of uncertainty. This confirms the importance of the choice of measure in portfolio optimization tasks. Another interesting continuation can be a study of the different results of the test portfolio using real-life data. This allows to determine a sum that will allow you to gain or lose by bringing the wrong shape for different values of the portfolio. Such a study seems more important for the investor and whether or with what amount of investment increased computational complexity is cost-effective.

\section{References}

[1] H. Markowitz, Portfolio Selection, The Journal of Finance, vol.7, no.1, 1952, pp.77-91.

[2] B. Liu and Y.-K. Liu, Expected value of fuzzy variable and fuzzy expected value models, Fuzzy Systems, IEEE Transactions on, vol. 10, no. 4,2002, pp. 445-450.

[3] J. Peng, H.M.K., Mok, T., Wai-Man, Credibility programming approach to fuzzy portfolio selection problems, Machine Learning and Cybernetics, 2005. Proceedings of 2005 International Conference on, vol.4, 2005, pp.2523-2528.

[4] X. Huang, Fuzzy chance-constrained portfolio selection, Applied Mathematics and Computation, vol. 177 , no. 2 , 2006, pp. 500-507.

[5] X. Huang, Mean-semivariance models for fuzzy portfolio selection, J.Comput. Appl. Math., vol. 217, no. 1, 2008, pp. 1-8.

[6] X. Huang, Mean-Entropy Models for Fuzzy Portfolio Selection, IEEE Transactions on Fuzzy Systems, vol. 16, 2008, pp. 1096-1101.

[7] X. Huang, Minimax mean-variance models for fuzzy portfolio selection, Soft Computing, vol. 15, no. 2,2010, pp. 251-260.

[8] X. Huang, Portfolio Analysis: From Probabilistic to Credibilistic and Uncertain Approaches, ser. Studies in Fuzziness and Soft Computing, Springer, 2010.

[9] X. Li, Z. Qin, and S. Kar, Mean-variance-skewness model for portfolio selection with fuzzy returns, European Journal of Operational Research, vol. 202, no. 1, 2010, pp. 239-247.

[10] P. Koprinkova, Membership functions shape and its influence on the dynamical behaviour of fuzzy logic controller, Cybernetics and Systems: An International Journal, vol. 2, no. 31,1952, pp. 161173.

[11] J. Marshall, M. Kazerani, and R. Shatshat, Investigation of membership function shapes in a 
fuzzy-controlled hvdc system, Industrial Electronics, 2006 IEEE International Symposium on, vol. 3, 2006, pp. 1800-1805.

[12] M. Multani, J. Ren, and V. Sood, Fuzzy logic (fl) controlled hvdc system-influence of shape ans distribution of membership functions (mfs) in Electrical and Computer Engineering (CCECE), 2010 23rd Canadian Conference on, 2010, pp. 1-7.

[13] B. Liu, Uncertainty Theory, ser. Studies in Fuzziness and Soft Computing. Springer, 2007.

[14] P. Li and B. Liu, Entropy of credibility distributions for fuzzy variables, Fuzzy Systems, IEEE Transac- tions on, vol. 16, no. 1, 2008 pp. 123-129.

[15] F. Wilcoxon, Individual comparisons by ranking methods, Biometrics Bulletin, vol. 1, no. 6, 1945, pp. 8083.

[16] S. Wang, J. Watada, and W. Pedrycz, Value-atRisk-Based Two-Stage Fuzzy Facility Location Problems, IEEE Transactions on Industrial Informatics, vol. 5, 2009, pp. 465-482.

[17] J. Peng, Measuring Fuzzy Risk by Credibilistic Value at Risk, in International Conference on Innovative Computing, Information and Control, 2008.

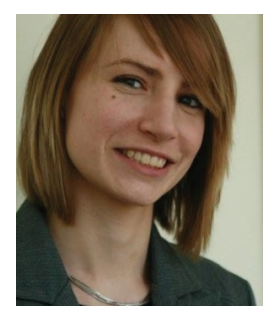

Aleksandra Rutkowska received a $\mathrm{Ph} . \mathrm{D}$. degree in economy from the Poznan University of Economics and Business, Poznan, Poland, in 2014. Since 2014 she has worked in the Department of Applied Mathematics. Her current research interests include portfolio optimization and preference modelling. She is a member of Polish Economic Society and the European Society for Fuzzy Logic and Technology. 